\title{
Hofstadter butterflies for flat bands
}

\author{
Hideo Aoki, Masato Ando* and Hajime Matsumura* \\ Department of Physics, University of Tokyo, Hongo, Tokyo 113, Japan
}

(October 25, 2018)

\begin{abstract}
Hofstadter's diagram, or the energy spectrum against the magnetic field in tight-binding systems, is obtained for the models having flat (dispersionless) one-electron band(s) that have originally been proposed for itinerant spin ferromagnetism. Magnetic fields preserve those flat bands that arise from a topological reason, while dispersions emerge in a singular manner for the flat bands arising from interference, implying an anomalous orbital magnetism.

PACS numbers: 72.15.Gd, 71.70.Di
\end{abstract}

Typeset using REVTEX 
Hofstadter's butterfly, or the Landau-quantized energy spectrum against the magnetic field in tight-binding systems, provides an intriguing example of fractal spectrum in the condensed matter physics. The quantum Hall effect for lattice fermions has also been discussed for the spectrum 1 . Physically, the message is that when the magnetic field penetrating the unit cell of a two-dimensional (2D) lattice is $q / p$ in units of flux quantum, we have essentially a $p$-band system. Accordingly the scaling of the integer quantum Hall effect, for instance, exhibits a peculiar structure for $p \neq 1$ 且.

Although the situation might seem essentially the same for complex lattices with the unit cell containing several atoms, here we wish to point out that an interesting physics does exist when there exist flat (dispersionless) band(s). The flat band, or a macroscopic number of degenerate states, has appeared in the condensed matter physics from various contexts.

First one concerns the spin magnetism in repulsively interacting itinerant electrons, as exemplified by the Hubbard model. It has become increasingly clear that only at, or possibly around, the singular limit of infinite interaction and infinitesimal doping from a half-filled band does a ferromagnetism appear. Lieb 3 then pointed out that we can realize a ferrimagnetism, for arbitrary strength of the Hubbard $U$ at half-filling, if a bipartite lattice with nearest-neighbor transfers has different numbers, $n_{\mathrm{a}} \neq n_{\mathrm{b}}$, of a and b sublattice sites in a unit cell. In this situation $n_{\mathrm{a}}-n_{\mathrm{b}}$ flat band(s) appear, so that the ferromagnetism resides on flat bands.

This is in accord with the 'generalized Hund's coupling 4 , which dictates that electrons on the Fermi surface should be fully spin-polarized for any $U$ - a macroscopic number of states lying on the Fermi energy will then imply a bulk magnetization. Curiously, each 'Wannier function' on the flat band cannot be confined to a unit cell contrary to a naive expectation that a dispersionless band should come from disjointed states. We can in fact identify the overlap of the Wannier states as an intuitive reason why the spins are ferromagnetically coupled 5 . This reminds us of the fractional quantum Hall system, where the quantum-liquid ground state is fully spin-polarized due to the exchange interaction among the orbitals in a Landau level, a peculiar 'flat band' arising itself from magnetic fields. There, orthogonalized 
'Wannier states' cannot be constructed either6.

The model is extended by Mielke 1 and by Tasaki 8 , which introduce distant-neighbor transfers to prepare flat band(s), on which spins align. Since the flat band is a result of an interference among the nearest-neighbor and more distant transfers, we may call this class the flat band due to interference. By contrast, Lieb's class may be called the flat band due to topology, since only the manner in which the sublattices are interlocked matters.

A class of flat bands has also been conceived in the context of 'lateral superstructures' that have superperiods of atomic dimensions along 2D directions. These are envisaged to be realized in organic $\pi$-electron materials such as the 'long-period graphite' (with period $\sim$ of a few tens of $\AA$ ), once alleged to be obtained in an attempt to fabricate fullerend. We can use the group theory 10 to classify all the atomic configurations with a superperiod into semiconducting, semimetallic and metallic classes. The superperiod such as super-honeycomb structures enforces, in some classes, the existence of flat bands on top of dispersive ones, which is a systematic realization of Lieb's case.

Now, a natural question is what will happen to the flat bands when a uniform magnetic field is applied. We can in fact expect intriguing phenomena, such as the orbital magnetism as in the 'ring-current effect' in fullerene 11 . In the present paper we show that the Hofstadter butterfly for the flat-band systems reveals that the magnetic field makes the flat bands remain flat, sandwiched between usual Hofstadter butterflies, for the flat bands arising from topology, while the flatness is lifted in a singular manner for the flat bands arising from interference. These imply that not only the spin magnetism but the orbital magnetism are intriguing in flat-band systems.

For the tight-binding model on complex lattices we consider for convenience a rectangular unit cell of $L_{x} \times L_{y}$ (which is twice the original unit cell in the case of honeycomb systems). The strength of the magnetic field, $B$, applied perpendicular to the system is characterized by $\tilde{B} \equiv B L_{x} L_{y} / \Phi_{0}=q / p$, where $\Phi_{0}=h / e$ is the flux quantum and the field is called rational when $p, q$ are integers.

The magnetic field is incorporated in the transfer energy, $t_{i j}$, in a usual manner through 
Peierls's phase as

$$
\begin{gathered}
t_{i j} \rightarrow e^{i \phi_{i j}} t_{i j}, \\
\phi_{i j}=-\frac{2 \pi}{\Phi_{0}} \int_{\mathbf{r}_{i}}^{\mathbf{r}_{j}} \mathbf{A} d \mathbf{r}=-\frac{2 \pi B}{\Phi_{0}} \bar{x}_{i j} \Delta y_{i j},
\end{gathered}
$$

where the last expression holds for the Landau gauge for the vector potential $\mathbf{A}=(0, B x)$.

In this gauge the phase appears for the transfer involving a shift along $x$, which repeats itself with a translation of the unit cells along $x$ by $N_{\text {cell }}$, where $N_{\text {cell }}$ is the smallest $N$ that makes $N(q / p)\left(\Delta y_{i j} / L_{y}\right)$ an integer for all the bonds $\langle i j\rangle$ within or across a unit cell. Thus we can perform a band-structure calculation regarding the $\left(N_{\text {cell }} L_{x}, L_{y}\right)$ system as a new unit cell. Its size depends by construction not only on $q / p$ but also on the atomic configuration in the original unit cell of the superstructure (via $\Delta y_{i j}$ ). In this respect the magnetic cell defined here differs from those appearing in the magnetic translation group. The existence of the cell implies that the Brillouin zone will be $N_{\text {cell }}$-folded.

Figure 1 displays the Hofstadter butterfly for simple realizations of Lieb's, Mielke's, and Tasaki's models, all assumed to have the square symmetry for simplicity. We can immediately see that, apart from the splitting of each dispersive band into magnetic minibands, the flat band in Lieb's case remains flat, whereas the interference-originated flat bands develop into peculiar butterflies as $B$ is increased.

The fact that the topological flat bands can evade the effect of $B$ is analytically shown. There we have only to solve three simultaneous eigenequations for three amplitudes $\psi_{A}, \psi_{B}, \psi_{C}$ (A at the vertex and $\mathrm{B}, \mathrm{C}$ at the mid points in the unit cell, depicted in Fig.1(a)). If we eliminate $\psi_{B}$ and $\psi_{C}$ the equation for nontrivial solutions for $\psi_{A}$ reduces to the corresponding equation for a simple square lattice if we translate $E_{\text {square }}$ into $E^{2}-4$. On top of these there is a class of $E=0$ states that have $\psi_{A} \equiv 0$, so that we have indeed a flat band with its energy pinned at the original energy that is literally sandwiched by two butterflies mapped via $\pm\left(E_{\text {square }}+4\right)^{1 / 2}$. Here the atomic level is taken to be $E=0$ (which coincides with $E_{F}$ when half-filled, i.e., one electron per atom) with $t=-1$. 
For $B=0$ the most compact 'Wannier state' (that cannot be orthogonalized as stressed) on the flat band is as depicted in inset of Fig.1(a). In quantum chemical language these states correspond, for finite molecules, to 'non-bonding molecular orbitals'. We can extend this by inspection to $B \neq 0$ as displayed in Fig.2. Curiously, the application of $B$ acts to deform the $E=0$ states into 'elongated ring states' along $x$ (or $y$ ) in the Landau gauge, whose length equals to $p$ for $\tilde{B}=q / p$. This sharply contrasts with the usual Bloch-Landau state having the size of the magnetic length $\propto 1 / \sqrt{B}$.

On the other hand, it is not surprising the flatness is lost even for an infinitesimal $B$ in Mielke's or Tasaki's models, which rely heavily on a exact tuning of the interference. If we look more closely at their Hofstadter butterflies, we can show some symmetries such as (i) a full periodicity is accomplished when the magnetic flux penetrating the smallest loop in the lattice becomes $\Phi_{0}$ (which is reminiscent of the $\mathrm{AB}$ effect in the mesoscopic conductance 2 , (ii) there is a two-fold symmetry about $\tilde{B}=1$ for Mielke's model or a mirror symmetry about $\tilde{B}=2$ for Tasaki's.

A more essential question is: can we identify the butterfly developed from the flat band with usual Landau's quantization? An indication that the situation is anomalous can be seen in Fig. 3, which displays how the 'Landau quantization' looks like for Tasaki's model. For $\tilde{B}=q / p$ with $p$ even, we have a series of Landau's bands (Harper-broadened Landau levels in a nonparabolic bands) that have a zero gap at the position, $E_{0}=-2$, of the original flat-band. For an odd $p$ the gap vanishes.

The density of states at $E_{0}$ thus alternates between zero and finite according to the parity of $p$, so that the orbital magnetic moment, $M=-\partial E_{T} / \partial B$ with $E_{T}$ being the total energy, becomes ill-defined along with the magnetic susceptibility. Another observation is that the density of states around $E_{0}$ spreads both below and above $E_{0}$ when $B$ is turned on, so that the total energy decreases when the magnetic flux is introduced if we start from a flat band less than half-filled. This might lead to an orbital ferromagnetism (a spontaneous induction of a network of 'persistent currents'), although it has been pointed out 13 in the context of the flux phase 14 in correlated electrons that a more accurate estimate of energy 
has to include the diamagnetic shift and the shrinkage of atomic orbitals.

We now turn to superhoneycomb systems, where in the classification by Shima and Aoki a class $\mathrm{B}_{0}\left(\mathrm{~B}_{C}\right)$ system has to have, when bipartite, at least three (one) flat band(s) in the gap of semiconducting (semimetallic) bands. To define the classes, we can first note that a unit cell in a honeycomb system may be regarded as comprising two atomic clusters (or 'superatoms'), where the two do not (case A) or have to (B) share an atom. The center of each superatom (a three-fold axis) may (case C) or may not (0) coincide with the position of an atom.

The result for the Hofstadter butterfly (Fig.4) shows that the flat bands remain flat for $B>0$ no matter whether they are non-degenerate $\left(\mathrm{B}_{C}\right)$ or degenerate $\left(\mathrm{B}_{0}\right)$ at $B=0$. For the system depicted in Fig.4(a) the flat band is sandwiched between the butterfly for the simple honeycomb lattice 15 just as in Fig.1(a), where the only difference is that the butterflies are now mapped via $\pm\left(E_{\text {honeycomb }}+3\right)^{1 / 2}$.

The presumed superstructures are surprisingly stable against the band Jahn-Teller type distortion as seen from the total energy calculation 16 . For actual fabrication, one possibility would be to polymerize self-aligned organic molecules as realized in the van der Waals epitaxy 17. Magneto-transport in these systems will be also of interest as in 3D organic materials 18

We thank Koichi Kusakabe, Kazuhiko Kuroki, and Naoto Nagaosa for valuable discussions. 


\section{REFERENCES}

* Present address: Institute for Solid State Physics, University of Tokyo, Roppongi, Tokyo 106, Japan.

${ }^{1}$ D.J. Thouless et al, Phys. Rev. Lett. 49, 405 (1982).

${ }^{2}$ H. Aoki, Surf. Sci. 263, 137 (1992).

${ }^{3}$ E.H. Lieb, Phys. Rev. Lett. 62, 1201 (1989).

${ }^{4}$ K. Kusakabe and H. Aoki, J. Phys. Soc. Japan 61, 1165 (1992); Phys. Rev. Lett. 72, 144 (1994).

${ }^{5}$ A. Mielke and H. Tasaki, Commun. Math. Phys. 158, 341 (1993).

${ }^{6}$ D.J. Thouless, J. Phys. C 17, L325 (1984).

${ }^{7}$ A. Mielke, J. Phys. A 24, 3311 (1991).

${ }^{8}$ H. Tasaki, Phys. Rev. Lett. 69, 1608 (1992).

${ }^{9}$ O.L. Chapman cited in H. Reiss and D.U. Kim, in Nonlinear Optical and Electroactive Polymers, ed. P.N. Prasad and D.R. Ulrich, (Plenum, 1988) p.281.

${ }^{10}$ N. Shima and H. Aoki, Phys. Rev. Lett. 71, 4389 (1993).

${ }^{11}$ V. Elser and R.C. Haddon, Nature 325, 792 (1987).

${ }^{12}$ C.P. Umbach et al, Appl. Phys. Lett. 50, 1289 (1987).

13 A. Alexandrov and H. Capellmann, Phys. Rev. Lett. 66, 365 (1991).

${ }^{14}$ Hasegawa et al, Phys. Rev. Lett. 63, 907 (1989); A.B. Harris et al, Phys. Rev. B 40, 2631 (1989).

${ }^{15}$ R. Rammal, J. Physique 46, 1345 (1985).

${ }^{16}$ H. Aoki and N. Shima, Superlattices and Microstructures 15, 247 (1994). 
${ }^{17}$ A. Koma in New Horizons in Low-Dimensional Electron Systems ed. by H. Aoki et al (Kluwer, Dordrecht, 1992), p.85.

${ }^{18}$ See, e.g., Y. Iye in High Magnetic Fields in the Physics of Semiconductors ed. by D. Heiman (World Scientific, Singapore, 1995), p.714. 


\section{Figure captions}

Fig.1 Hofstadter's diagram (energy spectrum against $\tilde{B}$ ) for Lieb's(a), Mielke's(b), and Tasaki's(c) models, whose lattice structures are attached with $t$ etc being the transfer. Arrows here and figures below represent the position of the flat bands for $B=0$, while a

'Wannier state' is encircled for Lieb's model. The spectrum are shown here for $\tilde{B} \equiv q / p$ with typically $p \leq 30$ or $1 \leq q \leq 119$ with $p=120$.

Fig.2 An example of the $E=0$ 'elongated ring states' in the Landau gauge, whose length equals to $p$ for $\tilde{B}=q / p(=1 / 5$ here $)$. A circle represents a finite amplitude, while arrows indicate the phase.

Fig.3 Typical band structures (projected onto $k_{x}$ plane or $k_{y}$ plane for finite numbers of k's) are displayed for $\tilde{B}=q / p$ with $p$ even $(q / p=1 / 10$, a $)$ or $p$ odd $(q / p=1 / 5, \mathrm{~b})$ in Tasaki's model. Note a change in the vertical scale between (a) and (b).

Fig.4 Hofstadter's diagram for class $\mathrm{B}_{0}(\mathrm{a})$ or $\mathrm{B}_{C}(\mathrm{~b})$ superhoneycomb systems. 